\title{
A Case of Autoimmune Hepatitis and Bisphosphonate-Related Osteonecrosis of the Jaw
}

\author{
Y.S. de Boer ${ }^{a} \quad$ G. Bouma ${ }^{a} \quad$ M.P. Wattjes ${ }^{b} \quad$ P. Lips ${ }^{c}$ \\ C.J.J. Mulder ${ }^{a}$ C.M.J. van Nieuwkerk ${ }^{a}$ \\ Departments of ${ }^{\mathrm{a}}$ Gastroenterology and Hepatology, ${ }^{\mathrm{b}}$ Radiology and \\ 'Endocrinology, VU University Medical Center, Amsterdam, The Netherlands
}

\section{Key Words}

Autoimmune hepatitis - Bisphosphonate - Osteonecrosis of the jaw $\cdot$ Complication

\begin{abstract}
Autoimmune hepatitis (AlH) is a chronic inflammatory liver disease of unknown aetiology usually requiring long-term immunosuppressive therapy. We present the case of an AlH patient who received long-term corticosteroids and azathioprine. As treatment for concomitant osteoporosis she was also treated with potent intravenous bisphosphonate (BP). This treatment was complicated by the development of BP-related osteonecrosis of the jaw (BRONJ). BRONJ is an uncommon complication of BP treatment regimes that occurs at increased frequency in the presence of other risk factors, including chronic inflammatory conditions. Our patient suffered from a severe and complicated clinical course of BRONJ which, despite adequate therapy, resulted in death of the patient. Here we discuss the risk factors for the development and clinical course of BRONJ in AlH and the implications for management of these patients.
\end{abstract}

\section{Introduction}

Autoimmune hepatitis (AIH) is a relatively uncommon chronic autoimmune liver disease of unknown aetiology. It can present at all ages and in both sexes, affecting women more frequently than men (4:1) [1]. The incidence and point prevalence of AIH in Northern Europe are 1.9 and 16.9 per 100,000, respectively [2]. The presentation of AIH ranges from asymptomatic liver test abnormalities to acute liver failure. AIH is characterised by hyperimmunoglobulinaemia and the presence of autoantibodies (smooth muscle antibodies, anti-nuclear antibodies, liver-kidney microsomal antibodies and/or soluble liver/liver-pancreas antibodies) in the serum [1]. Typical histological features in liver biopsy include interface hepatitis and plasmacellular 
infiltrates [1]. So far there is no single diagnostic test for AIH and due to the heterogenic presentation diagnosis may often be delayed in patients with otherwise unexplained transaminase abnormalities. In succession of the rather complex 1999 diagnostic AASLD criteria, a simplified scoring system was devised for practical and clinical use by Hennes et al. in 2008 [3, 4]. Untreated AIH has an overall 5-year mortality of $>50 \%$, due to development of cirrhosis-related oesophageal variceal bleeding and liver failure [1]. Induction therapy with initial high-dose corticosteroids is therefore warranted in a majority of patients, and this induces remission in up to $90 \%$ after 6 months [1]. A large proportion of patients with medication-induced remission requires long-term maintenance therapy because of high relapse rates upon tapering of corticosteroids. These regimens consist of either low-dose corticosteroids ( $5 \mathrm{mg} / \mathrm{day}$ ) or replacement therapy with azathioprine $(1-2 \mathrm{mg} / \mathrm{kg} /$ day) [1].

AIH patients are prone to develop osteoporosis, which is most likely due to several factors. These include prolonged corticosteroid exposure and, because of impaired synthetic hepatic function, high unconjugated bilirubin and impaired conversion of vitamin D to 25-hydroxy vitamin D [5, 6]. For this reason, bisphosphonate (BP) treatment is widely used in these patients [5]. A rare complication of this preventative form of treatment is BP-related osteonecrosis of the jaw (BRONJ) [7]. BRONJ is characterised by spontaneous occurrence of an osseous, necrotic lesion of either the mandible or maxilla in BP-treated patients. This results in an open connection of the necrotic lesion with the oral cavity [7]. We discuss here the case of an AIH patient who developed BRONJ with a particularly severe clinical course, which resulted in death of the patient.

\section{Case Report}

A 75-year-old woman presenting with decompensated liver cirrhosis was diagnosed with AIH. Although remission was initiated with prednisone and azathioprine therapy, liver function tests including bilirubin (28-61 $\mu \mathrm{mol} / \mathrm{l})$ remained elevated for more than 1 year after the start of treatment. Despite several tapering and replacement therapy attempts, she remained corticosteroid dependent ( $>7.5 \mathrm{mg} /$ day). At the time of diagnosis she underwent a bone mineral density (BMD) scan showing osteoporosis of the hip (BMD: $0.64 \mathrm{~g} / \mathrm{cm}^{2}$; T score: -2.5 ; z score: -0.9 ). To prevent further progression of osteoporosis, she was initially treated with intravenous pamidronate $60 \mathrm{mg}$ every 3 months, together with vitamin D and calcium supplementation. Initially this did not have an effect on BMD. However, her BMD increased when she was switched to once a year infusions of $5 \mathrm{mg}$ zoledronic acid.

After 3 years of intravenous BP therapy she developed malnutrition due to eating difficulties as a result of severe pain in the jaw. She was therefore admitted to our hospital. Examination of the jaw revealed that this was due to an infected necrotic mandibular bone, which had an open connection with the oral cavity. Computed tomography later confirmed multifocal osteonecrosis of the mandible (fig. 1, fig. 2). Given the BP use and the absence of any known other cause she was diagnosed with BRONJ and therefore subsequently treated with analgesics and prophylactic antibiotics. This did not prevent progression of the osteonecrotic infection, from which Pseudomonas aeruginosa and Candida albicans were cultured. She subsequently underwent repeated mandibular sequestrectomy and tooth extraction. Despite intense therapy she eventually succumbed to respiratory failure due to disseminated C. albicans pneumonia. 


\section{Discussion}

BRONJ development is mainly associated with long-term, high-dose intravenous $\mathrm{BP}$ regimens for the treatment of bone involvement of malignancies [7]. In these patients incidence rates up to $12 \%$ have been reported after more than 3 years of BP therapy, but it may occur already after 4 months in these patients [8, 9]. Following FDA approval, potent low-dose BP agents are increasingly used in the treatment of osteoporosis [7]. While the occurrence in this patient category is substantially lower, BRONJ can develop in these patients as our case demonstrates.

Treatment of symptomatic BRONJ lesions consists of analgesic therapy and antibiotics [7]. Depending on the severity of symptoms and the extension of the BRONJ lesions, debridement and sequestrectomy are indicated. These interventions generally give good results, but refractory cases have been described [7]. Discontinuation of intravenous BP therapy does not provide benefits in the short term, but may be beneficial in the long term [7]. Since food intake can be severely impaired by the inability to chew and swallow, adequate intake should be cared for to prevent malnutrition, if necessary by feeding through a nasogastric tube.

So far, the pathogenesis of BRONJ is incompletely understood. It has been proposed that BP therapy may contribute to the development of osteonecrosis of the jaw through the inhibition of osteoclast function, impaired angiogenesis and mucosal ulceration $[7,10,11]$. Furthermore, bacterial overgrowth and infection have been implicated in the development of BRONJ lesions [12]. The pro-inflammatory response against bacteria prevents healing of the lesion, which can again serve as an entry port for prolonged bacterial infection [12]. BP agents can also induce a pro-inflammatory immune response in BRONJ lesions, disrupting the mucosal barrier [12]. Autoimmune diseases like AIH show a similar inflammatory profile and may theoretically contribute to the development of these osteonecrotic lesions [12-14].

Immunosuppressive therapy as treatment for autoimmune diseases often includes systemic corticosteroids. Corticosteroid therapy itself increases the risk of osteonecrosis through vascular growth inhibition and osteoblastic apoptosis [13]. Therefore it is likely that corticosteroids may have an independent effect on the development of osteonecrosis of the jaw. Furthermore, immunosuppressive treatment increases the chances of oral bacterial overgrowth and thus may further increase the risk of infection of the jaw lesion. Concomitant use of immunosuppressants and potent $\mathrm{BP}$ agents therefore requires careful follow-up [7]. If BRONJ occurs, temporary discontinuation of immunosuppressants must be considered to enable the lesion to heal.

AIH may significantly contribute to BRONJ development in case of poor hepatic synthetic function, as high unconjugated and conjugated bilirubin levels are associated with decreased osteoblast viability and increased osteoclastogenesis [6]. The latter effect may be neutralized by potent BP agents, thus critically interfering with bone remodelling in the jaw.

Taken together, the above-mentioned factors may pose a particularly high risk for the development of BRONJ in patients with AIH. When starting BP treatment for the prevention of osteoporosis, we advise monitoring synthetic hepatic function and 
replacing corticosteroid therapy if possible. In case of BRONJ development, besides standard care, cessation of immunosuppressive therapy should be considered and the nutrition status should be guarded.
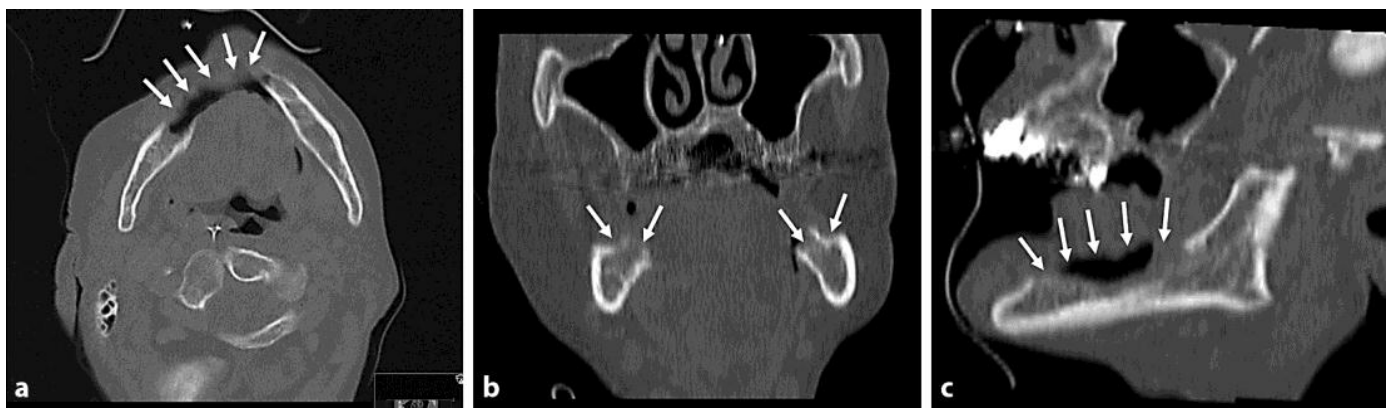

Fig. 1. Spiral computed tomography in the bone window setting of the mandible after sequestrectomy and tooth extraction. Note the bilateral cortical bone defects (arrows) suggestive of multifocal osteonecrosis in the mandibular body in the multiplanar reconstructions in axial (a), coronal (b) and sagittal (c) orientations.

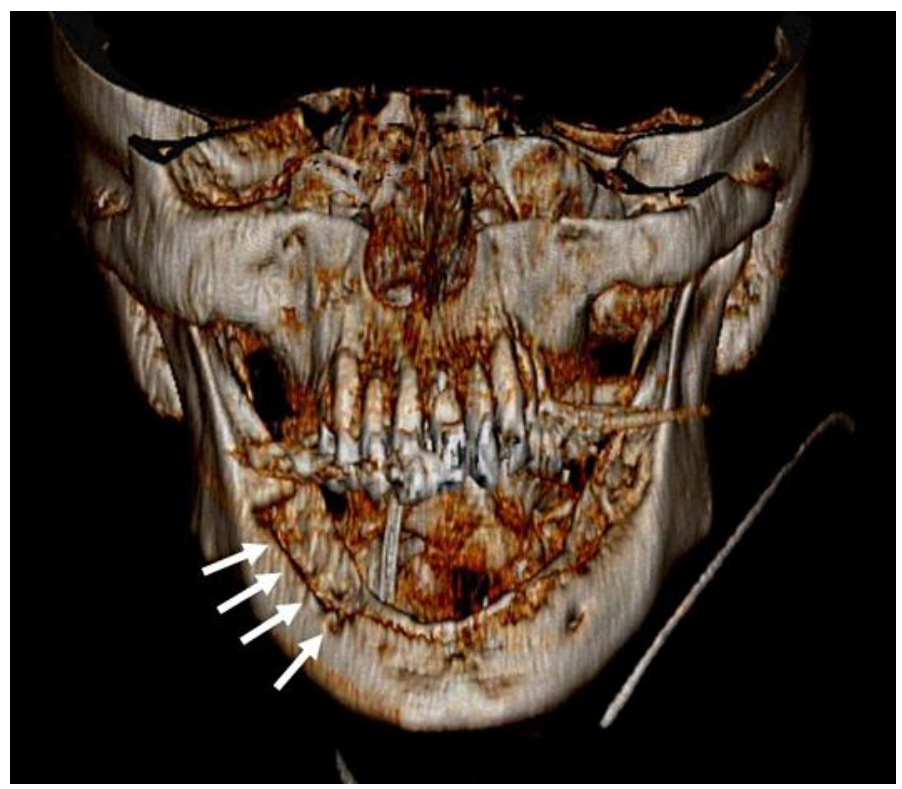

Fig. 2. Three-dimensional reconstruction of spiral computed tomography of the mandible using volume rendering technique. Note the extensive cortical defects of the mandible (arrows) suggestive of osteonecrosis. 


\section{References}

1 Manns MP, Czaja AJ, Gorham JD, Krawitt EL, Mieli-Vergani G, Vergani D, Vierling JM: Diagnosis and management of autoimmune hepatitis. Hepatology 2010;51:2193-2213.

$\checkmark 2$ Boberg KM, Aadland E, Jahnsen J, Raknerud N, Stiris M, Bell H: Incidence and prevalence of primary biliary cirrhosis, primary sclerosing cholangitis, and autoimmune hepatitis in a Norwegian population. Scand J Gastroenterol 1998;33:99-103.

-3 Alvarez F, Berg PA, Bianchi FB, Bianchi L, Burroughs AK, Cancado EL, Chapman RW, Cooksley WG, Czaja AJ, Desmet VJ, Donaldson PT, Eddleston AL, Fainboim L, Heathcote J, Homberg JC, Hoofnagle JH, Kakumu S, Krawitt EL, Mackay IR, MacSween RN, Maddrey WC, Manns MP, McFarlane IG, Meyer zum Buschenfelde KH, Zeniya M: International Autoimmune Hepatitis Group Report: review of criteria for diagnosis of autoimmune hepatitis. J Hepatol 1999;31:929-938.

-4 Hennes EM, Zeniya M, Czaja AJ, Pares A, Dalekos GN, Krawitt EL, Bittencourt PL, Porta G, Boberg KM, Hofer H, Bianchi FB, Shibata M, Schramm C, Eisenmann de Torres B, Galle PR, McFarlane I, Dienes HP, Lohse AW: Simplified criteria for the diagnosis of autoimmune hepatitis. Hepatology 2008;48:169-176.

5 Watts NB, Bilezikian JP, Camacho PM, Greenspan SL, Harris ST, Hodgson SF, Kleerekoper M, Luckey MM, McClung MR, Pollack RP, Petak SM; AACE Osteoporosis Task Force: American Association of Clinical Endocrinologists Medical Guidelines for Clinical Practice for the diagnosis and treatment of postmenopausal osteoporosis. Endocr Pract 2010;16(suppl 3):1-37.

-6 Ruiz-Gaspa S, Martinez-Ferrer A, Guanabens N, Dubreuil M, Peris P, Enjuanes A, Martinez de Osaba MJ, Alvarez L, Monegal A, Combalia A, Pares A: Effects of bilirubin and sera from jaundiced patients on osteoblasts: contribution to the development of osteoporosis in liver diseases. Hepatology 2011;54: 2104-2113.

7 Ruggiero SL, Dodson TB, Assael LA, Landesberg R, Marx RE, Mehrotra B; American Association of Oral and Maxillofacial Surgeons: American Association of Oral and Maxillofacial Surgeons position paper on bisphosphonate-related osteonecrosis of the jaws - 2009 update. J Oral Maxillofac Surg 2009;67:2-12.

-8 Durie BG, Katz M, Crowley J: Osteonecrosis of the jaw and bisphosphonates. N Engl J Med 2005;353: 99-102.

-9 Abu-Id MH, Warnke PH, Gottschalk J, Springer I, Wiltfang J, Acil Y, Russo PA, Kreusch T: 'Bis-phossy jaws' high and low risk factors for bisphosphonate-induced osteonecrosis of the jaw. J Craniomaxillofac Surg 2008;36:95-103.

10 Van den Wyngaert T, Huizing MT, Vermorken JB: Bisphosphonates and osteonecrosis of the jaw: cause and effect or a post hoc fallacy? Ann Oncol 2006;17:1197-1204.

11 Marx RE: Pamidronate (Aredia) and zoledronate (Zometa) induced avascular necrosis of the jaws: a growing epidemic. J Oral Maxillofac Surg 2003;61:1115-1117.

12 Compston J: Pathophysiology of atypical femoral fractures and osteonecrosis of the jaw. Osteoporos Int 2011;22:2951-2961.

13 Zalavras C, Shah S, Birnbaum MJ, Frenkel B: Role of apoptosis in glucocorticoid-induced osteoporosis and osteonecrosis. Crit Rev Eukaryot Gene Expr 2003;13:221-235.

14 Mehanna P, Goddard R: Bisphosphonate associated osteonecrosis: an unusual case. Aust Dent J 2010;55: 311-313. 